\title{
Optimizing natural gas fueling station reservoirs pressure based on ideal gas model
}

\author{
Mahmood Farzaneh-Gord, Mahdi Deymi-Dashtebayaz* \\ The Faculty of Mechanical Engineering, Shahrood University of Technology, Shahrood, Iran \\ "Corresponding author: meh_deimi@yahoo.com
}

\begin{abstract}
At CNG fuelling station, natural gas is usually stored in a cascade storage system to utilize the station more efficient. The cascade storage system is generally divided into three reservoirs, commonly termed low, medium and high-pressure reservoirs. The pressures within these three reservoirs have huge effects on the performance of a CNG fuelling station and a fast filling process of natural gas vehicle's (NGV) cylinder. A theoretical analysis is developed to study the effects of the reservoirs pressures and temperatures on the performance of the CNG station. The analysis is based on the first and the second law of thermodynamics, conservation of mass and ideal gas assumptions. The results show that as the reservoir temperature decreases, the fill ratio increases and the pressure within the filling station reservoirs has no effects on the fill ratio. The non-dimensional entropy generation and filling time profiles have opposite trends and as entropy generation decreases, the filling time increases. The optimized non-dimensional low and medium pressure-reservoir pressures are found to be as 0.24 and 0.58 respectively in thermodynamic point of view.
\end{abstract}

Keywords: Compressed natural gas, CNG cylinder, Fast-filling process, Cascade Reservoirs, Thermodynamic analysis, Entropy generation.

\section{INTRODUCTION}

Compressed natural gas (CNG) is used as a clean alternative to other automobile fuels such as gasoline (petrol) and diesel ${ }^{1}$. Although there are huge available resources of natural gas, it has not been widely accepted as an alternative fuel to gasoline by most countries. The main obvious reason is low driving range of natural gas vehicles (NGV) which is partly associated with the natural gas fuelling stations technology and the filling process.

The natural gas vehicles (NGV) usually receive natural gas from high pressure reservoirs at a fuelling station during the filling. The first problem with the NGV fuel stations is the refuelling time of an NGV. The NGV industry has made excellent advancements in the industry to provide a system to refuel a NGV in a comparable to that of a gasoline dispenser. The problem with the long refuelling time has been remedied for the most part to be comparable to the filling time $(<5 \mathrm{~min})$ taken to fill a gasoline powered automobile. This $(<5 \mathrm{~min})$ filling time can be referred to as a fast filling or rapid charge.

The on-board storage capacity of natural gas vehicles is another problem to the wide spread marketing of these alternate fuelled vehicles. The on-board storage cylinders encountered a rise in storage gas cylinder temperature (in the range of $40 \mathrm{~K}$ or more ${ }^{2}$ ) during the fast filling due to complex compression and mixing processes. This temperature rise reduces the density of the gas in the cylinder, resulting in an under-filled cylinder, relative to its rated specification. If this temperature rise is not compensated for in the fuelling station dispenser, by transiently over-pressurizing the tank, the vehicle user will experience a reduced driving range. Although the NGV on-board cylinder volume plays the main role in on-board storage capacity, the fuelling station reservoirs pressure has also big effects on the amount of the filled mass of the on-board cylinder.

Gas from the distribution pipeline is compressed using a large multi-stage compressor into a "cascade" storage system. The input work required by the compressor is the final problem with the fuelling station as the required work for compressing and storing the natural gas is then partially wasted through the filling process.

In order to make the utilization of the natural gas fuelling station more efficient, natural gas is usually stored in a cascade storage system. The cascade storage system is commonly divided into three reservoirs, generally termed low, medium and high-pressure reservoirs. It could be proved that the pressure within the low, medium and high-pressure reservoirs have big effects on 3 problems associated with the filling process and the fuelling station as below:

1) Filling time;

2) The charged mass of the on-board cylinder after refuelling;

3) The compressor input work.

Considering the above 3 problems with a fuelling station, one could conclude increasing the filled mass of the on-board cylinder, the performance of the fuelling station could be improved.

To understand the fast filling process and study the effect of the pressure and temperature within the low, medium and high-pressure reservoirs on the performance of a fuelling station, a theoretical analysis has been developed based on the first law of thermodynamics, conservation of mass and ideal gas assumptions in this study. The fast filling process was assumed to be a quasi-static process and the natural gas presumed to be purely Methane (as an ideal gas). The second law analysis has been employed to calculate the amount of entropy generation during filling process. It is well known that less entropy generation is associated with less required work by the compressor.

To the authors knowledge, there have been no previous researches on performance enhancement of a fuelling station and the filling process. But, there have been limited researches in the field of filling process modelling in the literature. Kountz ${ }^{2}$ was the first who modelled the fast filling process of natural gas storage cylinder based 
on the first law of thermodynamics. They developed a computer program to model the fast filling process for a single reservoir for real gas. Kountz et al. ${ }^{3-6}$, have also developed a natural gas dispenser control algorithm that insures a complete filling of the NGV cylinders under a fast filling scenario. The researchers are also under way to model the fast filling of hydrogen-based fuelling infrastructure including the work of Liss and Richards ${ }^{7}$, Liss et al. ${ }^{\mathbf{2}}$, Farzaneh-Gord et al. ${ }^{\mathbf{9}}$ and Newhouse and Liss $^{\mathbf{1 0}}$ have studied the fast filling of hydrogen cylinder using a number of experiments. They reported a high temperature increase in the cylinder during the process.

A few experimental studies were also carried out to study the fast filling of a natural gas cylinder including the work of Thomas and Goulding ${ }^{11}$ and Shipley ${ }^{\mathbf{1 2}}$. Shipley concluded that ambient temperature change can have an effect on the fast fill process. He also concluded that the test cylinder was under-filled every time it was rapidly recharged.

Farzaneh et al. ${ }^{13}, 14$ have also modelled the fast filling process. They developed a computer program based on Peng-Robinson state equation and methane properties table for a single reservoir. They investigated the effects of ambient temperature and initial cylinder pressure on the final cylinder conditions. In another study, Farzaneh et al. ${ }^{15}$ presented the thermodynamics analysis of the cascade reservoirs filling process of natural gas vehicle cylinders. The results of this research indicated that ambient temperature has a big effect on the filling process and the final NGV cylinder conditions.

Farzaneh-Gord et al. ${ }^{\mathbf{1 6}}$ have employed a theoretical analysis to study the effects of buffer and cascade storage banks on the performance of a CNG fuelling station. It has been found that the time (filling time) required for bringing up the $\mathrm{NGV}$ onboard cylinder to its final pressure in the buffer storage system is about $66 \%$ less than that of the cascade storage bank. The charged mass for cascade bank is about $80 \%$ of the buffer bank which gives an advantage to buffer bank over cascade one. The biggest advantage of the cascade bank over the buffer is $50 \%$ less entropy generation for this configuration, which probably causes much lower required compressor input work for this configuration comparing to buffer bank.

Farzaneh-Gord et al. ${ }^{17,18}$ have also studied the effects of natural gas compositions on the fast filling process for the buffer and cascade storage banks. In these researches the conditions of storage banks were considered constant. For studying the CNG filling process, it is vital to know the thermodynamic properties of natural gas. To compute the thermodynamic properties, the AGA8 Equation of State has been employed. The AGA8 equation of state has been developed by American Gas Association, especially for calculating the compressibility factor and density of natural gas for custody transfer.

As mentioned above, the second law has been employed in this study to theoretically calculate the amount of the entropy generation. Entropy generation is associated with thermodynamic irreversibilities, which is common in all types of thermal systems. Various sources are accountable for the entropy generation. There have been numerous researches in the field of entropy generation. Bejan $^{19,20}$ has concentrated upon the different mechanisms responsible for entropy generation in applied thermal engineering. Generation of entropy destroys the available work of a system. Therefore, it makes a good engineering sense to focus on irreversibilities (see Bejan 19-21) of heat transfer and fluid flow processes and try to understand the function of related entropy generation mechanisms. Since then, a lot of investigations have been carried out to compute the entropy generation and irreversibility profiles for different geometric configurations, flow situations, and thermal boundary conditions. Here, entropy generation minimization has been employed as an objective function for the optimization the $\mathrm{CNG}$ fuelling process.

\section{THE CNG FILLING STATION}

Figure 1 shows a typical CNG filling station. Gas from the distribution pipeline, usually "low" pressure at $<0.4 \mathrm{MPa}$ or possibly "medium" pressure $(<1.6 \mathrm{MPa})$, is compressed using a large multi-stage compressor into a "cascade" storage system. It should be pointed out that in some European countries, the term low and medium pressure may stand for another pressure range. This system is maintained at the pressure higher than that in the vehicle's on-board storage so that gas flows to the vehicle under differential pressure. Typically, the cascade storage will operate in the range of $20.5 \mathrm{MPa}$ to $25 \mathrm{MPa}$, while the vehicle's maximum onboard cylinder pressure is 200 bar. In order to make the utilization of the compressor and buffer storage more efficient, the fast filling CNG stations usually operate using a three-stage "cascade" storage system.

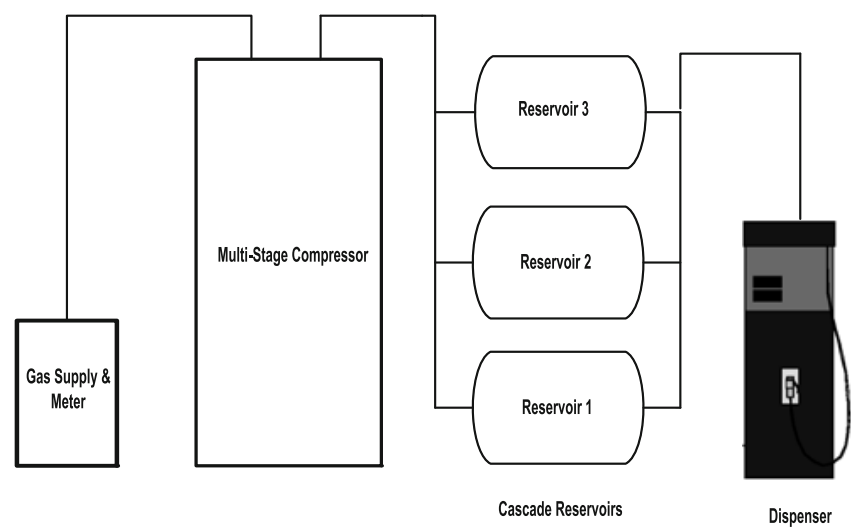

Figure 1. A schematic diagram of the NGV Filling Station

The cascade storage system is usually divided into three reservoirs, commonly termed low, medium and high-pressure reservoirs. During fast filling, the onboard cylinder is first connected to the low-pressure reservoir. As the pressure in the reservoir falls and that in the on-board cylinder rises, the flow of gas decreases. When the flow rate has declined to a pre-set level the system switches to the medium pressure reservoir, then finally to the high-pressure reservoir to complete the fill. It is expected that the cascade system results in a more complete "fill" than if the whole buffer storage were maintained at one pressure and utilises the compressor and storage with maximum efficiency. In addition, when the compressor is automatically switched on to refill the reservoirs it fills the high pressure reservoir first, then switches to the medium and the low reservoirs. This ensures that the high, pressure reservoir (used to com- 
plete the fill) is maintained at maximum pressure all the time, ensuring that vehicles are always supplied with the maximum amount of gas available. Correct specification of the compressor capacity and the volume of cascade storage is necessary to ensure that the CNG station can cope with the type (LGVs, buses or trucks) and frequency (peak periods) of vehicles using the facility.

\section{COMPRESSED NATURAL GAS CYLINDERS}

The natural gas cylinders have various design types based on the used construction materials. The design types include ${ }^{22}$ Type 1 , which are all-metal, Type 2, which have a metal liner and hoop wrapped composite reinforcement, Type 3, which have a metal liner and a full wrapped composite reinforcement, and Type 4, which have a non-metallic liner and a full wrapped composite reinforcement. Metal containers and liners are typically steel or aluminium. Composite reinforcements are typically carbon or glass fibbers in an epoxy resin matrix. CNG cylinders are designed for a specified nominal service pressure at a specified temperature essentially a specified density $\left(\mathrm{kg} / \mathrm{m}^{3}\right)$ of fuel. This will result in a given mass of natural gas stored in the fuel container. The actual pressure in the fuel container will vary from the nominal service pressure as the temperature of the fuel in the container varies. Under-filling of NGV cylinders, during fast fill charging operations, can occur at fuelling stations, at ambient temperatures greater than $30^{\circ} \mathrm{C}$. The resulting reduced driving range of the vehicle is a serious obstacle which the gas industry is striving to overcome, without resorting to unnecessarily high fuelling station pressures, or by applying extensive over-pressurization of the cylinder during the fuelling operation. Undercharged storage cylinders are a result of the elevated temperature which occurs in the NGV storage cylinder. Fuelling stations normally fill the cylinder up $125 \%$ of nominal service pressure to avoid under charging but this highly depends to ambient temperature.

\section{CASCADE RESERVOIRS PARAMETERS}

There are a few important parameters affecting the filling process which should firstly be introduced.

Figure 2 shows a schematic diagram of cascade reservoirs which has been under investigation in this study. Thermodynamic properties in cascade reservoirs play important roles on filling process. Two main thermodynamic properties are pressure and temperature. As shown in Fig. 2, each reservoir has its own temperature $\left(T_{R}\right)$ and pressure $\left(\mathrm{P}_{\mathrm{R}}\right)$ which assumed unchanged while the pressure and temperature within on-board NGV cylinder varies during the filling process. Typically, the maximum pressure in high-pressure reservoir $\left(\mathrm{P}_{\mathrm{R} 3}\right)$ is in the range of $20.5 \mathrm{MPa}$ to $25 \mathrm{MPa}$, while the vehicle's maximum onboard cylinder pressure is $20 \mathrm{MPa}$.

To maintain the final pressure within the onboard cylinder high enough, the pressure within high-pressure reservoir assumed constant (20.5 MPa) throughout this study. The effects of medium and low reservoirs pressures on the performance of the fuelling station have been studied by introducing the two dimensionless parameters. The ratio of medium and low pressures to high-pressure one which defined as below, are these two parameters:

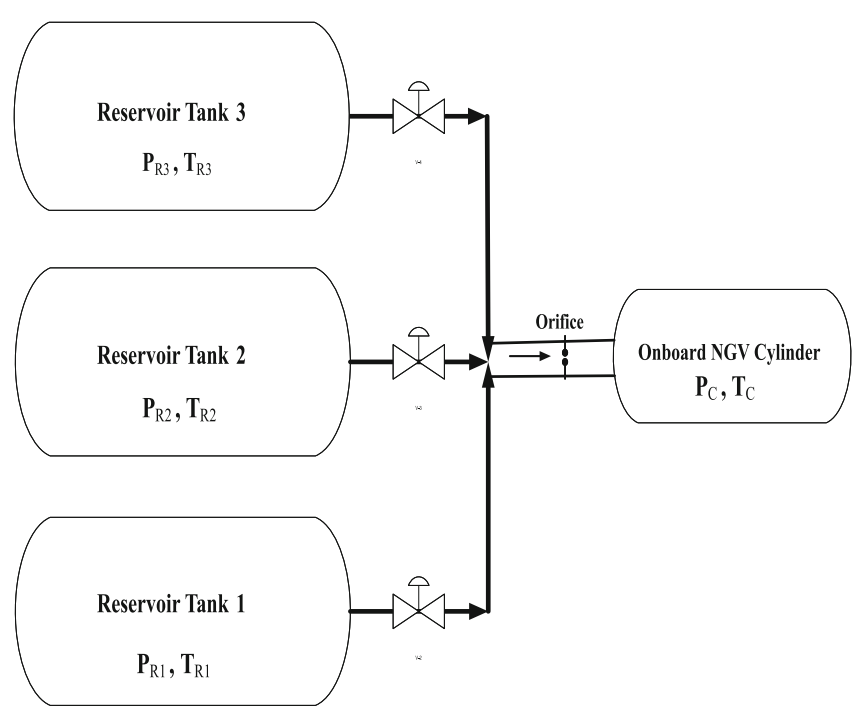

Figure 2. A schematic diagram of cascade reservoirs

$N P 1=P_{R 1} / P_{R 3} \quad N P 2=P_{R 2} / P_{R 3}$

The initial on-board cylinder pressure also affects the fuelling station performance. Here the dimensionless on-board cylinder pressure, $N P_{C}$, and on-board cylinder initial pressure, $N P_{C i}$, are defined as follows:

$N P_{C}=P_{C} / P_{R 3} \quad N P_{C i}=P_{C i} / P_{R 3}$

It should be pointed out that the high-pressure reservoir pressure kept constant at $20.5 \mathrm{MPa}$ throughout this study.

The non-dimensional in-cylinder and ambient temperature could also be defined to study the effects of reservoir, cylinder and ambient temperatures on filling process as follows:

$N T_{C i}=T_{C i} / T_{R} \quad N T_{C}=T_{C} / T_{R} \quad N T a=T_{\infty} / T_{R}$

In which $T_{R}$ is the temperature of natural gas compressed in (all reservoirs assumed to have same temperature) reservoirs, $T_{C i}$ is the initial temperature of the on-board cylinder and $T_{\infty}$ is the ambient temperature. In this study ambient temperature is fixed at $300 \mathrm{~K}$.

The final dimensionless number is the "fill ratio". The cylinder "fill ratio" is defined as the mass of charged gas after refuelling divided by the mass which the cylinder could hold at the rating condition $(300 \mathrm{~K}, 200 \mathrm{bar})$. This parameter is directly related to the driving range of the NGV and defined as:

$F R=\frac{m_{c}(\text { at end of filling })}{\rho(300 \mathrm{~K}, 200 \mathrm{bar}) V_{c}}$

\section{THERMODYNAMIC ANALYSIS}

\section{First law analysis}

In this study to model the fast filling process and develop a mathematical method, the NGV on-board cylinder is considered as a thermodynamic open system which goes through a quasi-steady process.

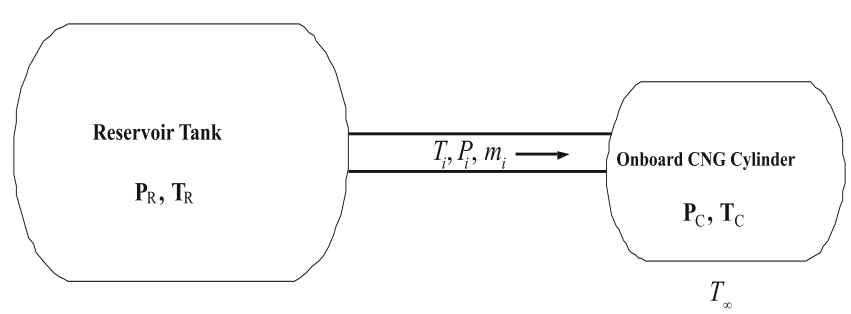

Figure 3. A schematic diagram of thermodynamic system 
To develop a theoretical analysis, the continuity and first law of thermodynamics has been applied to the cylinder to find two thermodynamics properties. Considering the onboard NGV cylinder as a control volume and knowing it has only 1 inlet, the continuity (conservation of mass) equation may be written as follows:

$\frac{d m_{C}}{d t}=\dot{m}_{i}$

In equation $5, \dot{m}_{i}$ is the inlet mass flow rate and can be calculated by considering an isentropic expansion through an orifice. Applying gas dynamics laws:

$\dot{m}_{i}=C_{d} \rho_{R} A_{\text {orifice }}\left(\frac{P_{C}}{P_{R}}\right)^{\frac{1}{\gamma}}\left\{\left(\frac{2 \gamma}{\gamma-1}\right)\left(\frac{P_{R}}{\rho_{R}}\right)\left[1-\left(\frac{P_{C}}{P_{R}}\right)^{\frac{\gamma-1}{\gamma}}\right]\right\}$

if $\frac{P_{C}}{P_{R}} \leq\left(\frac{2}{\gamma+1}\right)^{\frac{\gamma}{\gamma-1}}$

$\dot{m}_{i}=C_{d} \rho_{R} A_{\text {orifice }}\left(\frac{P_{C}}{P_{R}}\right)^{\frac{1}{\gamma}}\left\{\left(\frac{2 \gamma}{\gamma-1}\right)\left(\frac{P_{R}}{\rho_{R}}\right)\left[1-\left(\frac{P_{C}}{P_{R}}\right)^{\frac{\gamma-1}{\gamma}}\right]\right\}^{\frac{1}{2}}$

if $\frac{P_{C}}{P_{R}}>\left(\frac{2}{\gamma+1}\right)^{\frac{\gamma}{\gamma-1}}$

In equations $6,7 C_{d}$ the coefficient of the orifice is discharged. It could be considered as a factor which brings non-ideality of the gas into accounts.

The First laws of thermodynamics for a control volume in a general form can be written as follows:

$\dot{Q}_{c v}+\sum \dot{m}_{i}\left(h_{i}+\frac{V_{i}^{2}}{2}+g z_{i}\right)=\sum \dot{m}_{e}\left(h_{e}+\frac{V_{e}^{2}}{2}+g z_{e}\right)+\frac{d}{d t}\left(m\left(u+\frac{V^{2}}{2}+g z\right)\right)_{c v}+\dot{W}_{c v}$

The work term is zero in the filling process and the change in potential and kinetic energy can be neglected. The equation then could be simplified as below:

$\frac{d U_{C}}{d t}=\delta \dot{Q}+\dot{m}_{i}\left(\frac{V_{i}^{2}}{2}+h_{i}\right)$

As $h_{R}=\frac{V_{i}^{2}}{2}+h_{i}$, the above equation further could be

simplified as:

$\frac{d U_{C}}{d t}=\delta \dot{Q}+\dot{m}_{I} h_{R}$

The heat lost from the on-board NGV cylinder to environment could be calculated as

$\delta \dot{Q}=-U_{H C} A_{C}\left(T_{C}-T_{\infty}\right)$

Combining 5, 10 and 11, one could calculate the above equation as:

$\frac{d\left(m_{C} u_{C}\right)}{d t}=-U_{H C} A C\left(T_{C}-T_{\infty}\right)+\frac{d m_{C}}{d t} h_{R}$

Or in the following form:

$\frac{d\left(m_{C} u_{C}\right)}{d t}-\frac{d\left(m_{C} h_{R}\right)}{d t}=-U_{H C} A_{C}\left(T_{C}-T_{\infty}\right)$

The above equation could be rearranged in the following form:

$d\left(m_{C} u_{C}-m_{C} h_{R}\right)=-U_{H C} A_{C}\left(T_{C}-T_{\infty}\right) d t$

The above equation could be integrated from the "start" of filling, up to the "current" time as:

$\int_{s}^{c} d\left(m_{C} u_{C}-m_{C} h_{R}\right)=-\int_{o}^{t} U_{H C} A_{C}\left(T_{C}-T_{\infty}\right) d t$

The integration of the above equation for a single reservoir fuelling station resulted in:
$m_{C}\left(u_{C}-h_{R}\right)-m_{C s}\left(u_{C s}-h_{R}\right)=-U_{H C} A_{C} \Delta T_{a v} t$

When $m_{c}, m_{c s}$ are mass of the charged gas at the "current" and the "start" of the filling process, $\Delta T_{a v}$ is an average temperature difference between the cylinder and environment which defined as:

$\Delta T_{a v}=\frac{1}{t} \int_{o}^{t}\left(T_{C}-T_{\infty}\right) d t$

The first law of thermodynamics for the on-board NGV cylinder could finally be written as:

$u_{C}=h_{R}-U_{H c} A_{C} \Delta T_{a v} t+\frac{m_{C s}}{m_{C}}\left(u_{C s}-h_{R}\right)$

Equations 5, 6 and 18 could be employed to calculate the two thermodynamics properties of in-cylinder natural gas at any time. By knowing two thermodynamics properties (here specific internal energy and specific volume), the other in-cylinder properties could be found.

\section{ADIABATIC SYSTEM}

For an adiabatic system, equation 18 could be more simplified as:

$u_{C}=h_{i}+\frac{m_{C s}}{m_{C}}\left(u_{C s}-h_{R}\right)$

And if $m_{c s}=0$, the following relation is valid at any time:

$u_{c}=h_{r}$

\section{IDEAL GAS MODEL FOR ADIABATIC SYSTEM}

For the case of assuming an ideal gas behaviour, the governing equation could be much simplified. Considering the following ideal gas assumptions:

$u=c_{v} T, h=c_{p} T, m=\frac{P V}{R T}$

And knowing that the volume of the cylinder, specific heats, reservoir temperature are constant, then equation 15 could be simplified as follows:

$d(m u)_{c v} / d t=\dot{m}_{i} h_{R} \rightarrow d\left(P V / R T \times c_{v} T\right)_{c v} / d t=\dot{m}_{i} c_{p} T_{R} \rightarrow V_{c v} c_{v} / R \times d\left(P_{c v}\right) / d t=\dot{m}_{i} c_{p} T_{R}$

By replacing the inlet mass flow rate from equation 6 and 7 , the following simple equation could be obtained:

$d\left(P_{C}\right) / d t=\dot{m}_{i}\left(\gamma R / V_{c v}\right) T_{r}=\left\{\begin{array}{l}\left(\gamma R / V_{c v}\right) T_{R} C_{d} \rho_{R} A_{\text {orifice }}\left(\frac{P_{C}}{P_{R}}\right)^{\frac{1}{\gamma}}\left\{\left(\frac{2 \gamma}{\gamma-1}\right)\left(\frac{P_{R}}{\rho_{R}}\right)\left[1-\left(\frac{P_{C}}{P_{R}}\right)^{\frac{\gamma-1}{\gamma}}\right]\right\}^{\frac{P_{C}}{P_{R}}} \leq\left(\frac{2}{\gamma+1}\right)^{\frac{\gamma}{\gamma-1}} \\ \left(\gamma R / V_{c v}\right) T_{R} C_{d} \sqrt{\gamma P_{R} \rho_{R}} A_{\text {orifice }}\left(\frac{2}{\gamma+1}\right)^{\frac{\gamma+1}{2(\gamma-1)}} \\ \text { if } \frac{P_{C}}{P_{R}}>\left(\frac{2}{\gamma+1}\right)^{\frac{\gamma}{\gamma-1}}\end{array}\right.$

\section{The Second law analysis}

The second law of thermodynamics and flow processes occurring in the "cascade" storage system of the CNG filling station, adopted in this study, makes it possible to evaluate the entropy generation rates, $\dot{S}_{g e n}$, for the characteristic nodes of the system.

The second law of thermodynamics for the filling process of an on-board NGV cylinder could be presented as:

$\dot{S}_{g e n}=d S_{C} / d t-\delta \dot{Q} / T_{\infty}-\dot{m}_{i} s_{i} \geq 0$

Here, all irreversibility assumed to be occurred from the inlet to the in-cylinder position. This makes an isentropic expansion from the reservoir to the inlet position, 
which means $s_{i}=s_{R}$. Considering this assumption and combining equations 5, 11 and 24, the following equation could be obtained:

$\dot{S}_{\text {gen }}=\frac{d\left(m_{C} s_{C}\right)}{d t}-\frac{d m_{C}}{d t} s_{R}+U_{H c} A_{C}\left(T_{C}-T_{\infty}\right) / T_{\infty}$

Or in the following form:

$\dot{S}_{g e n} d t=d\left(m_{C} s_{C}-m_{C} s_{R}\right)+U_{H C} A_{C}\left(T_{C}-T_{\infty}\right) / T_{\infty} d t$

The above equation could be integrated from the "start" of the filling to the "current" time as below:

$S_{g e n}=\int_{s}^{c} d\left(m_{C} s_{C}-m_{C} s_{R}\right)+\int_{s}^{c} \frac{U_{H C} A_{C}\left(T_{C}-T_{\infty}\right)}{T_{\infty}} d t$

For a fuelling station with a single reservoir in which $s_{R}$ remains constant throughout the filling process, the integration of the above equation resulted in a simple equation as:

$S_{g e n}=m_{C}\left(s_{C}-s_{R}\right)-m_{C s}\left(s_{C s}-s_{R}\right)+\frac{U_{H C} A_{C}\left(T_{a v}-T_{\infty}\right)}{T_{\infty}}$

\section{ADIABATIC SYSTEM}

Equation 28 could be more simplified for an adiabatic system as:

$S_{\text {gen }}=m_{C}\left(s_{C}-s_{R}\right)-m_{C s}\left(s_{C s}-s_{R}\right)$

And if the cylinder is empty at start of the filling process $\left(m_{c s}=0\right)$ the following relation could be obtained: $S_{\text {gen, } \max }=m_{C}\left(s_{C}-s_{R}\right)$

\section{IDEAL GAS MODEL FOR ADIABATIC SYSTEM}

For the case of assuming an ideal gas behavior, the second law could be much simplified. Considering the following ideal gas assumptions:

$s_{C}-s_{R}=c_{p} \ln \frac{T_{C}}{T_{R}}-R \ln \frac{P_{C}}{P_{R}}, m_{c}=\frac{P_{C} V_{C}}{R T_{C}}$

And knowing that the volume of the cylinder, $V_{c}$, specific heat, $c_{p}$, reservoir temperature are constant then equation 29 could be simplified as follows:

$S_{\text {gen }}=m_{C}\left(c_{p} \ln \frac{T_{C}}{T_{R}}-R \ln \frac{P_{c}}{P_{R}}\right)-m_{C s}\left(c_{p} \ln \frac{T_{C s}}{T_{R}}-R \ln \frac{P_{C s}}{P_{R}}\right)$

For the case where $m_{c s}=0$ and assuming the pressure within the on-board cylinder reaches its reservoir pressure $\left(p_{c} \approx p_{R}\right)$, then equation 32 gives te maximum entropy generation for a fuelling station as follows:

$S_{\text {gen, } \max }=m_{C} c_{p} \ln \frac{T_{c}}{T_{R}}$

Considering equation 20 and 21, the above equation could be more simplified as follows:

$S_{g e n, \max }=\frac{c_{v} P_{R} V_{c}}{R T_{R}} \ln \gamma$

It should be noted that equation 31 to 34 are only valid for a single reservoir fuelling station. Calculating entropy generation for the fuelling station with cascade reservoirs system demands more efforts. Here the non-dimensional entropy generation has been introduced to compare the results for various configurations as follows: $N_{S}=S_{\text {gen }} / S_{\text {gen,max }}$

It is worth mentioning that $N S$ expresses irreversibility in the system. The minimizing NS means reducing work destruction in the system ${ }^{\mathbf{1 9 - 2 0}}$. As all work required by the system is provided by the station compressor, one can conclude that minimum NS indicates the least input work by the compressor.

\section{The numerical procedure}

The procedure for computing the in-cylinder properties begins by knowing the initial conditions (pressure and temperature). The other initial thermodynamics properties (including $h_{R}=u_{R}+(p v)_{R}$ ) are calculated by employing Ideal gas equation discussed in the previous section. Equation 6 (or 7) is employed to calculate the inlet mass flow rate. Equation 5 is then employed to compute the in-cylinder mass and consequently specific volume within the cylinder using first order Euler numerical scheme. Similarly, equation 23 is solved to calculate the in-cylinder pressure at the new time step. Upon determination of the pressure and specific volume, the temperature could be found from the ideal gas law. The entropy generation then could be calculated from equation 32 .

\section{RESULTS AND DISCUSSION}

In this study, the NGV cylinder has been considered adiabatic as a result, the characteristics of the orifice, will not affect the final temperature state in the cylinder. The orifice diameter and the cylinder volume were considered to be $1 \mathrm{~mm}$ and 67 Litres respectively. The results have been presented here for a commonly used cascade group of three as shown in Fig. 2.

For the results presented here, it is assumed that the natural gas obeys ideal gas law. It is instructive to evaluate the discrepancy of the natural gas from ideal gas model. Figure 4 shows variation of density of methane for real and ideal gas models for an average filling temperature. As shown in this figure, the discrepancy of density is small at the low pressure and rises as pressure increases. There is about $20 \%$ density discrepancy at maximum pressure. As, the ideal gas model gives a high degree of simplicity, it may be justified to use the model for the CNG filling station process.

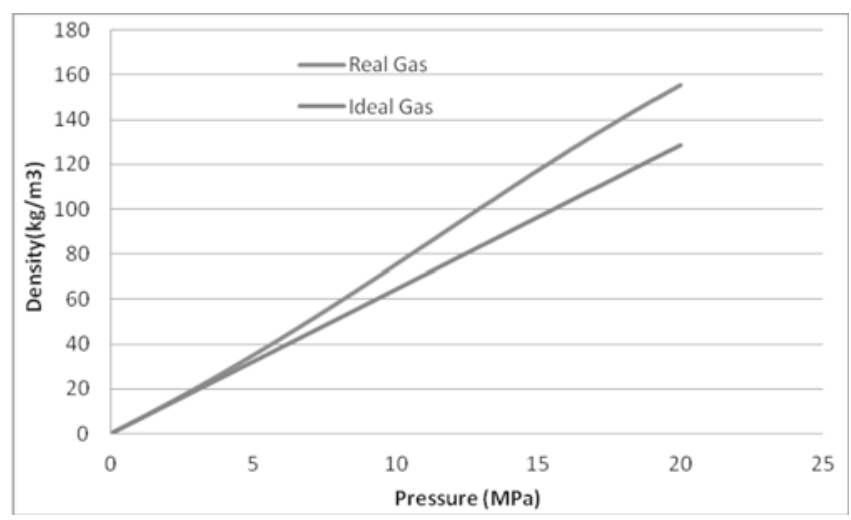

Figure 4. Variation of methane density in average filling temperature for both ideal and real gas

Figure 5 shows the effects of non-dimensional initial cylinder pressure on non-dimensional dynamic pressure profiles during filling. Note from the figure, as the non-dimensional initial cylinder pressure increases, the filling time decreases. Discontinuity in pressure profile is due to switching to another reservoir tank.

Figure 6 shows the effect of initial cylinder temperature on the dynamic temperature profiles of NGV cylinder, 


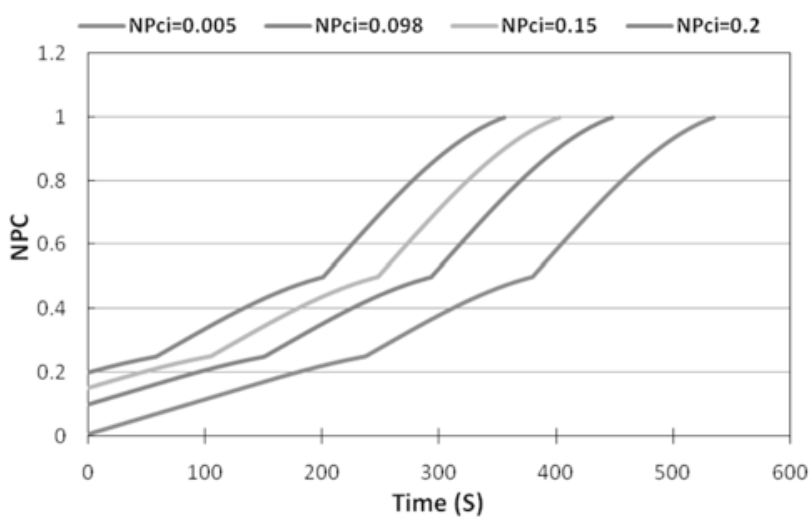

Figure 5. Effect of initial cylinder pressure on dynamic in-cylinder pressure

during filling which could represent ambient temperature effect. Note form figure, the cylinder gas temperature rises sharply during early charging time and flattens after. It should be pointed out that, Farzaneh et al. ${ }^{\mathbf{1 5}}$ have studied filling process of cascade reservoirs using real gas model. They have obtained similar trends for the dynamic in-cylinder pressure profiles. For the temperature profile, they found two dips in the temperature profile due to Joule-Thomson cooling effects when supply reservoir change.

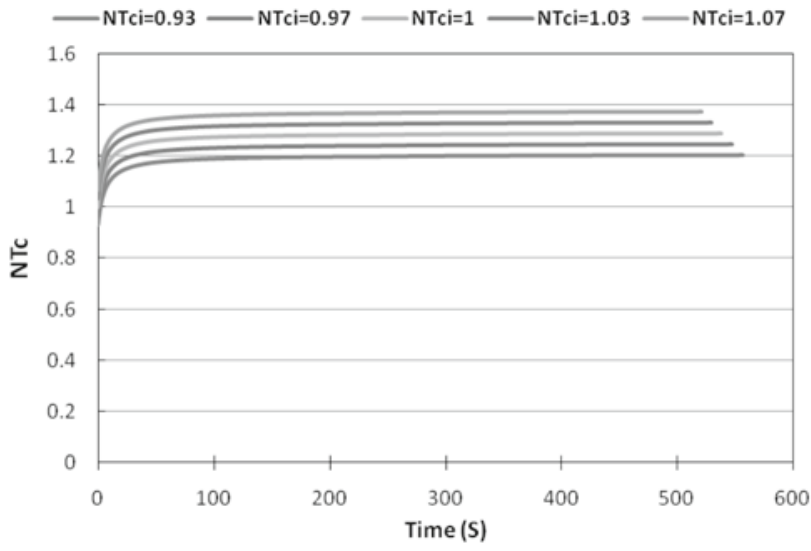

Figure 6. Effect of reservoir temperature on dynamic in-cylinder temperature

Figure 7 shows how the fill ratio varies with the initial temperature (in NGV cylinder) which could represent the effect of ambient temperature. It can be seen as the initial temperature increases the fill ratio decreases. This means that the driving range of an NGV will probably decrease for hot weather compared to the colder conditions. The same conclusion can be made by studying the effect of ambient temperature on the charged mass of NGV cylinder in same figure. By noting from the figure, the final in-cylinder mass decreases as ambient temperature increases. It should be pointed out that for an ideal gas model, $h_{R}$ is only a function of the reservoir temperature and so, the final in-cylinder temperature and pressure (and the fill ratio) remain unchanged as the low or medium reservoir pressure varies.

Figure 8 shows the effects of low pressure-reservoir pressure on the filling time while medium-pressure pressure is kept constant $(N P 2=0.525)$ for various non-dimensional initial pressure. It could be realised that the filling time increases as the initial in-cylinder

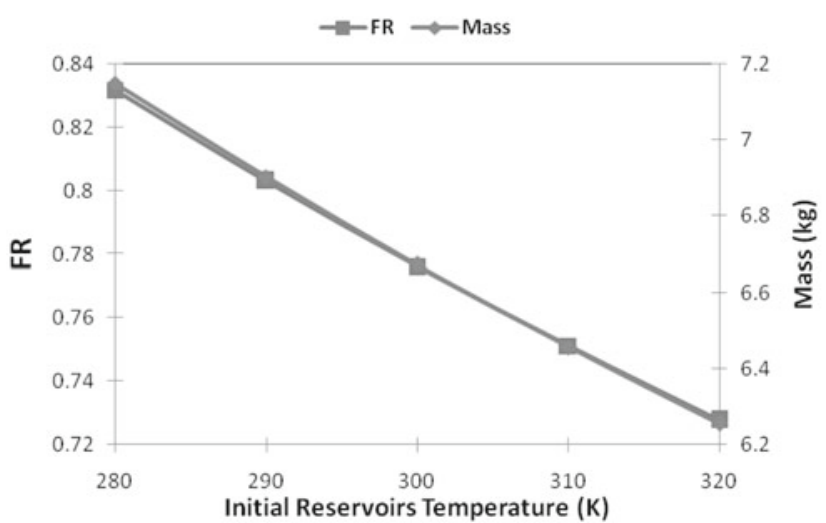

Figure 7. Effect of the initial reservoirs temperature on the fill ratio and the amount of charged gas

pressure. This is due to more fill up required. The maximum filling time occurs for an empty cylinder $\left(N P_{C i}=\right.$ 0.005 ) when $N P 1$ approaches 0.12 but generally depends on the initial in-cylinder pressure.

Figure 9 shows the effects of medium pressure-reservoir pressure on the filling time while the low-pressure pressure is kept constant $(N P 1=0.275)$ for various non-dimensional initial pressure. The same conclusion as Figure 7 could be made except that the maximum filling time doesn't depend on the initial in-cylinder pressure and occurs around 0.55 .

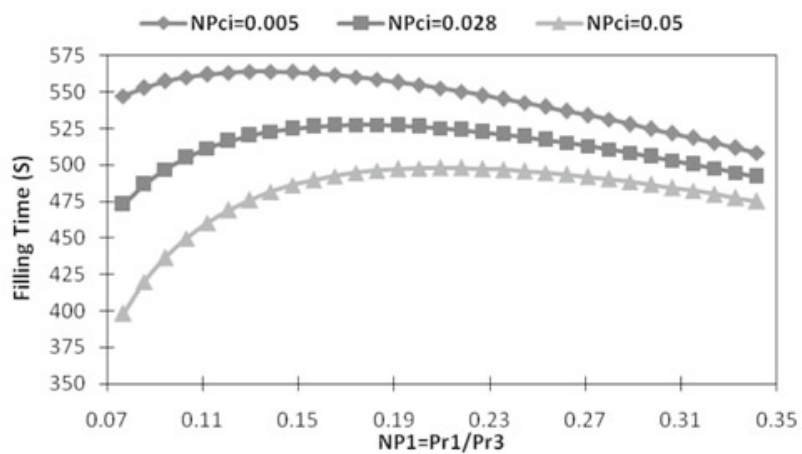

Figure 8. Effect of the varying low-pressure reservoir pressure $(N P 1)$ and the initial cylinder pressure $\left(N P_{C i}\right)$ on the filling time $(N P 2=0.525, N T a=1)$

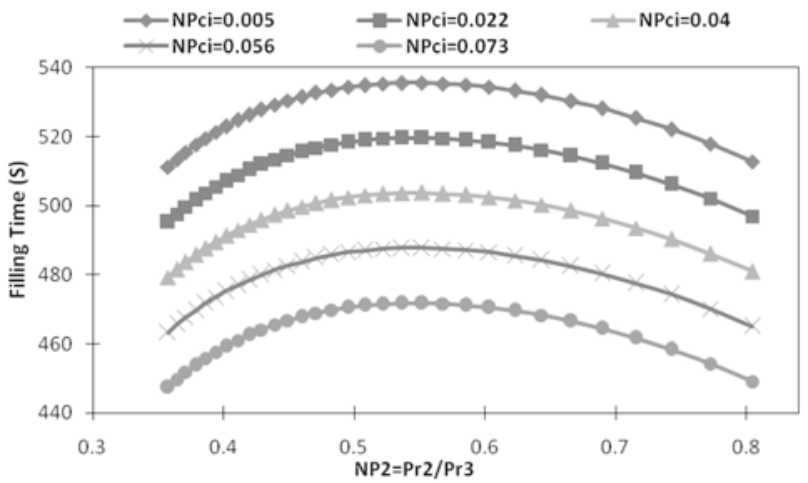

Figure 9. Effect of the varying medium-pressure reservoir pressure $(N P 2)$ and the initial cylinder pressure on the filling time $(N P 1=0.275, N T a=1)$

Figure 10 shows the effects of low-pressure reservoir pressure on the filling time while medium-pressure reservoir pressure is kept constant $(N P 2=0.525)$ for various non-dimensional reservoirs temperature. It could be realized as reservoirs temperature increases, 
the filling time decreases. This is due to the fact that the in-cylinder pressure rises to its final value faster for higher temperature inlet compared to lower values and the fact that filling stops as pressure receives its set point (20 MPa here). Note from the figure that the maximum filling time occurs at $N P 1 \approx 0.12$ for all cases.

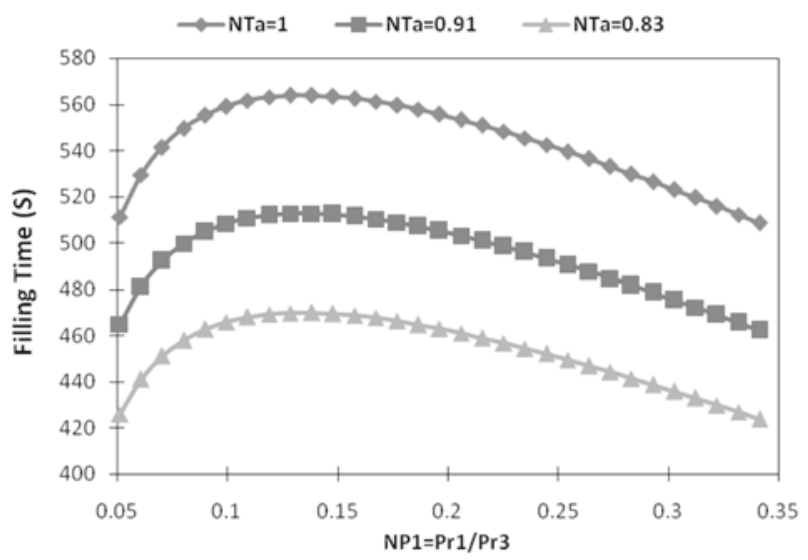

Figure 10. Effect of the varying low-pressure reservoir pressure $(N P 1)$ and the initial cylinder temperature $(N T a)$ on the filling time $\left(N P 2=0.525, N P_{C i}=0.005\right)$

Figure 11 shows the effects of low and medium pressure-reservoir pressure on the filling time of an empty cylinder $\left(N P_{C i}=0.005\right)$. Considering the fact that lessening the filling time is a way to enhance a filling station performance, a designer should seek for a combination of NP1 an NP2 in which the filling time is minimized. Note from the figure that the filling time increases as $N P 1$ decreases. For any value of $N P 1$, there is a specific value of $N P 2$ in which the filling time is maximized. So one could conclude that no such combination of $N P 1$, NP2 could be found.

It should also be noted that the filling time could also be reduced by appropriate sizing of piping equipments (e.g. orifice diameter).

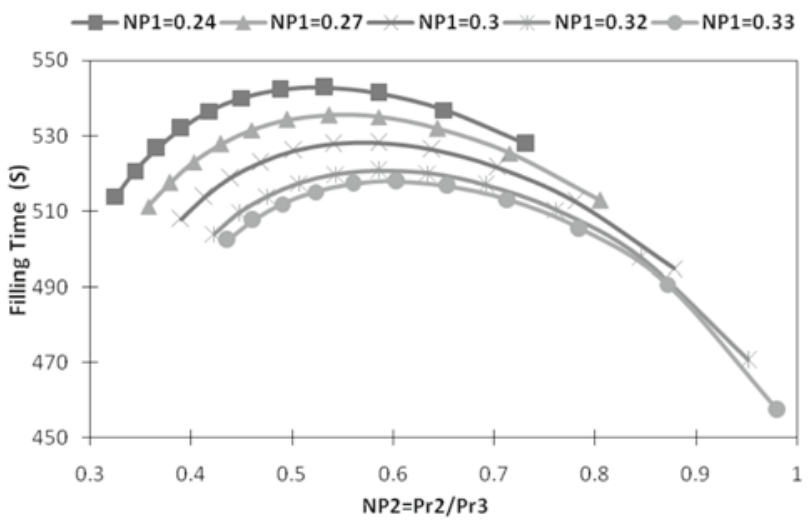

Figure 11. Effect of the varying low-pressure $(N P 1)$ and the medium pressure reservoir pressure $(N P 2)$ on the filling time $\left(N P_{C i}=0.005, N T a=1\right)$

As mentioned previously, Entropy generation is associated with thermodynamics irreversibilities. Irreversibilities destroy available work in the filling station. As, the available work is provided by the compressor so one could conclude that as entropy generation is decreased, available work destruction is decreased, too.
Figure 12 shows the effects of low-pressure reservoir pressure on non-dimensional entropy generation while medium-pressure pressure is kept constant $(\mathrm{NP} 2=0.525)$ for various non-dimensional initial pressure. It could be realised that the non-dimensional entropy generation decreases as the initial in-cylinder pressure increases. The minimum entropy generation occurs for $0.19<\mathrm{NP} 1$ $<0.27$.

Figure 13 shows the effects of medium-pressure reservoir pressure on the non-dimensional entropy generation while low-pressure pressure kept constant $(\mathrm{NP} 2=0.275)$ for various non-dimensional initial pressure. It could be realised that the non-dimensional entropy generation decreases as the initial in-cylinder pressure increases. The minimum entropy generation occurs for NP2 $\approx 0.58$.

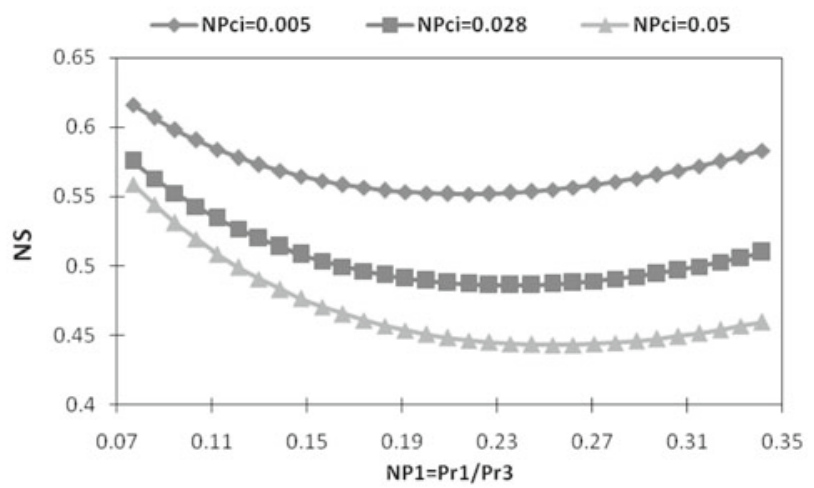

Figure 12. Effect of the varying low-pressure reservoir pressure $(N P 1)$ and the initial cylinder pressure $\left(N P_{C i}\right)$ on the non-dimension entropy generation $(N P 2=0.525$, $N T a=1)$

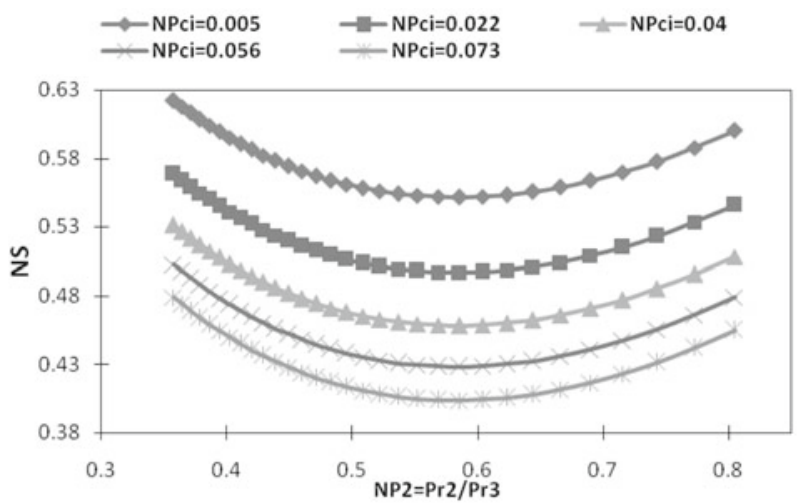

Figure 13. Effect of the varying medium-pressure reservoir pressure $(N P 2)$ and the initial cylinder pressure $\left(N P_{C i}\right)$ on the non-dimension entropy generation $(N P 1=0.275$, $N T a=1)$

Figure 14 shows the effects of low-pressure reservoir pressure on the non-dimensional entropy generation while medium-pressure reservoir pressure is kept constant $(N P 2=0.525)$ for various non-dimensional reservoirs temperature. It could be realized that the reservoirs temperature has very small effects on the non-dimensional entropy generation.

Figure 15 shows the combined effects of low and the medium pressure-reservoir pressure on the non-dimensional entropy generation of an empty cylinder $\left(N P_{C i}=\right.$ $0.005)$. Note from the figure that the minimum entropy generation occurs while $N P 2 \approx 0.58$ and $N P 1 \approx 0.24$. Considering the fact that most of NGV on-board cylinders are refuelled when they are empty, one could conclu- 


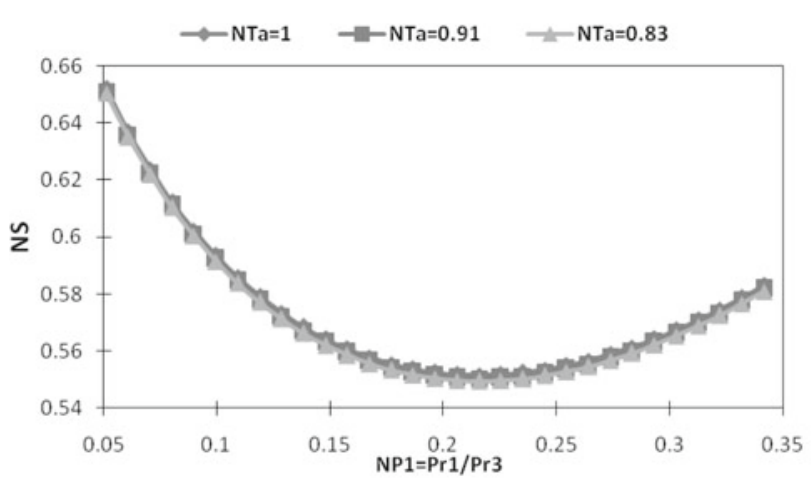

Figure 14. Effect of the varying low-pressure reservoir pressure $(N P 1)$ and the initial cylinder temperature $(N T a)$ on the non-dimension entropy generation $(N P 2=0.525$, $\left.N P_{C i}=0.005\right)$

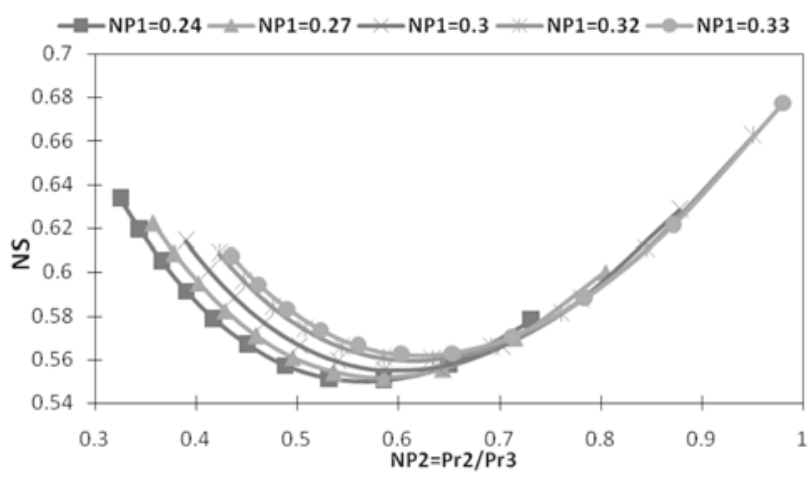

Figure 15. Effect of the varying low-pressure $(N P 1)$ and the medium-pressure reservoir pressure (NP2) on the non-dimension entropy generation $\left(N P_{C i}=0.005, N T a=1\right)$

de that optimized low and medium pressure-reservoir pressures are as the above from the thermodynamics point of view.

Considering Figs. 8-11 and $12-15$, it could be realized that the non-dimensional entropy generation and the filling time profiles have opposite trends. So as entropy generation in the system decreases, the filling time increases. As the filling time could be reduced by appropriate sizing of piping equipments, one could conclude that the optimized thermodynamics point could be selected to enhance the performance of a fuelling station.

\section{CONCLUSIONS}

The First and Second Laws of Thermodynamics have been employed as theoretical tools to enhance the performance of a cascade reservoir natural gas fuelling station. A theoretical analysis has been built to study the effects of reservoirs temperature and pressure on the fill ratio, the filling time and entropy generation during the fat fill process of the on-board NGV cylinder.

It is found that as the reservoir temperature decreases, the fill ratio increases and pressure within the filling station reservoirs has no effects. This is due to assuming ideal gas assumptions in this study.

Non-dimensional entropy generation and the filling time profiles have opposite trends and as entropy generation in the system decreases, the filling time increases. As the filling time could be reduced by appropriate sizing of piping equipments, one could conclude the optimized thermodynamics point could be selected for enhancing the performance of a fuelling station.
The optimized non-dimensional low and medium pressure-reservoir pressures are found to be as $N P 1 \approx 0.24$ and $N P 2 \approx 0.58$ from the thermodynamics point of view.

\section{NOMENCLATURE}

\begin{tabular}{|c|c|}
\hline A & area, $\left(\mathrm{m}^{2}\right)$ \\
\hline $\mathrm{C}_{\mathrm{d}}$ & orifice discharge coefficient \\
\hline$c_{p}, c_{v}$ & $\begin{array}{l}\text { Constant pressure \&volume specific heats, } \\
(\mathrm{kj} / \mathrm{kg} \mathrm{K})\end{array}$ \\
\hline$g$ & Gravitational acceleration, $\left(\mathrm{m} / \mathrm{s}^{2}\right)$ \\
\hline$h$ & Specific enthalpy, (kj/kg) \\
\hline$\dot{m}$ & Mass flow rate, $(\mathrm{kg} / \mathrm{s})$ \\
\hline M & Molecular weight, $(\mathrm{kg} / \mathrm{kmol})$ \\
\hline$P$ & Pressure, (bar or $\mathrm{Pa})$ \\
\hline$Q$ & Heat transfer rate, $(\mathrm{kW})$ \\
\hline$T$ & Temperature, $\left(\mathrm{K}\right.$ or $\left.{ }^{\circ} \mathrm{C}\right)$ \\
\hline$u$ & Internal energy, $(\mathrm{kj} / \mathrm{kg})$ \\
\hline $\mathrm{h}$ & Enthalpy, $(\mathrm{kj} / \mathrm{kg})$ \\
\hline $\mathrm{s}$ & Entropy, $(\mathrm{kj} / \mathrm{K})$ \\
\hline $\mathrm{t}$ & time, $(\mathrm{S})$ \\
\hline$v$ & Specific volume, $\left(\mathrm{m}^{3} / \mathrm{kg}\right)$ \\
\hline$V$ & Volume, $\left(\mathrm{m}^{3}\right)$ \\
\hline W & Actual work, (kj/kg) \\
\hline$\dot{W}$ & Actual work rate, (kW or MW) \\
\hline$z$ & Height, (m) \\
\hline$\rho$ & Density, $\left(\mathrm{kg} / \mathrm{m}^{3}\right)$ \\
\hline$N T$ & Temperature Ratio \\
\hline$N P$ & Pressure Ratio \\
\hline$\gamma$ & Isentropic Exponent \\
\hline
\end{tabular}

\section{Subscript}

C NGV on-board cylinder

$\mathrm{R} \quad$ reservoir tank

i initial or inlet condition

$\mathrm{s} \quad$ start of filling process

a, $\infty$ ambient

av average

gen generation

1 Reservoir tank 1

2 Reservoir tank 2

3 Reservoir tank 3

\section{LITERATURE CITED}

1. Mansha M., Saleemi A.R., Javed S.H. \& Nadeem Feroz (2011). Analysis of a Detailed Kinetic Model of Natural Gas Combustion in IC Engine, Polish Journal of Chemical Technology, 13, 1, 6-15, DOI: 10.2478/v10026-011-0002-0.

2. Kountz, K. (1994). Modeling The Fast Fill Process in Natural Gas Vehicle Storage Cylinders", American Chemical Society Paper at 207th National ACS Meeting, March.

3. Kountz, Kenneth J. \& Blazek, Christopher F. (1997). NGV Fuelling Station and Dispenser Control Systems," report GRI97/0398, Gas Research Institute, Chicago, Illinois, November.

4. Kountz, K., Liss, W. \& Blazek, C. (1998). Method and Apparatus For Dispensing Compressed Natural Gas", U.S. Patent 5,752,552, May 19.

5. Kountz, K., Liss, W. \& Blazek, C. (1998). Automated Process and System For Dispensing Compressed Natural Gas", U.S. Patent 5,810,058, Sept. 22.

6. Kountz, K., Liss, W. \& Blazek, C. (1998) A New Natural Gas Dispenser Control System, Paper at 1998 International Gas, Research Conference, San Diego, November 3. 
7. Liss, W.E. \& Richards M. (2003). Development of a Natural Gas to Hydrogen Fueling Station, Topical Report for U.S. DOE, GTI-02/0193, Sept., 2002.

8. Liss, W.E., Richards, M.E., Kountz, K. \& Kriha, K. (2003) "Modeling and Testing of Fast-Fill Control Algorithms for Hydrogen Fueling," 2003 National Hydrogen Association Meeting, March.

9. Farzaneh-Gord, M., Deymi-Dashtebayaz, M., Rahbari, HR. \& Nyazmand H. (2012) Effects of storage types and conditions on compressed hydrogen fuelling stations performance. International Journal of Hydrogen energy, 37, 3500-3509, DOI: 10.1016/j.bbr.2011.03.031.

10. Newhouse, N.L. \& Liss, W.E. (1999). Fast Filling of NGV Fuel Containers, SAE paper 1999-01-3739.

11. Thomas, G., Goulding, J. \& Munteam, C. ( 2002). Measurement, "Approval and Verification of CNG Dispensers", NWML KT11 Report.

12. Shipley, E. (2002). Study of natural gas vehicles (NGV) during the fast fills process", Thesis for Master of Science, College of Engineering and Mineral Resources at West Virginia University.

13. Farzaneh-Gord, M., Eftekhari, H., Hashemi, S., Magrebi, M. \& Dorafshan, M. (2007). The effect of initial conditions on filling process of CNG cylinders", The second International conference on Modeling, Simulation, And Applied optimization, Abu Dhabi, UAE, March 24-27.

14. Farzaneh-Gord, M. (2008). Compressed natural gas Single reservoir filling process", Gas international Engineering and Management, Volume 48, Issue 6, July/August, pp 16-18.

15. Farzaneh-Gord, M, Hashemi, SH, Farzaneh-Kord, A. (2008). Thermodynamics Analysis of Cascade Reserviors Filling Process of Natural Gas Vehicle Cylinders, World Applied Sciences Journal, 5 (2): 143-149.

16. Farzaneh-Gord, M., Deymi-Dashtebayaz, M. \& Rahbari, H.R. (2011). Studying effects of storage types on performance of CNG filling stations, Journal of Natural Gas Science and Engineering, Vol. 3, 334-340. DOI: 10.1016/j.jngse.2011.02.001.

17. Farzaneh-Gord, M., Deymi-Dashtebayaz, M. \& Rahbari, H.R. 2012. Effects of Natural Gas Compositions on CNG Fast Filling Process for Buffer Storage System, Oil \& Gas Science and Technology - Rev. IFP Energies nouvelles.

18. Farzaneh-Gord, M., Deymi-Dashtebayaz, M. \& Rahbari, H.R. 2012. Optimizing Compressed Natural Gas Filling Stations Reservoir Pressure Based on Thermodynamic Analysis, Int. J. Exergy, Vol. 10, No. 3. DOI: 10.1504/IJEX.2012.046836.

19. Bejan, A. (1982) Second-law analysis in heat transfer and thermal design, Adv. Heat Transfer 15, 1-58.

20. Bejan, A. (1996). Entropy Generation Minimization, CRC, Boca Raton, NY.

21. Bejan, A. (1979). A study of entropy generation in fundamental convective heat transfer, J. Heat Transfer 101, 718-725.

22. Wise Gas: CNG Cylinder Safety, http://www.wisegasinc. com/wg-cylindersafety.htm 